\title{
Effect of remote ischemic preconditioning on cognitive function after off-pump coronary artery bypass graft: a pilot study
}

\author{
Kyoung-Woon Joung, Jin-Ho Rhim, Ji-Hyun Chin, Wook-Jong Kim, Dae-Kee Choi, Eun-Ho Lee, \\ Kyung-Don Hahm, Ji-Yeon Sim, and In-Cheol Choi \\ Department of Anesthesiology and Pain Medicine, Asan Medical Center, University of Ulsan College of Medicine, Seoul, Korea
}

Background: Several studies have shown in animal models that remote ischemic preconditioning (rIPC) has a neuroprotective effect. However, a randomized controlled trial in human subjects to investigate the neuroprotective effect of rIPC after cardiac surgery has not yet been reported. Therefore, we performed this pilot study to determine whether rIPC reduced the occurrence of postoperative cognitive dysfunction in patients who underwent off-pump coronary artery bypass graft (OPCAB) surgery.

Methods: Seventy patients who underwent OPCAB surgery were assigned to either the control or the rIPC group using a computer-generated randomization table. The application of rIPC consisted of four cycles of $5 \mathrm{~min}$ ischemia and $5 \mathrm{~min}$ reperfusion on an upper limb using a blood pressure cuff inflating $200 \mathrm{mmHg}$ before coronary artery anastomosis. The cognitive function tests were performed one day before surgery and again on postoperative day 7 . We defined postoperative cognitive dysfunction as decreased postoperative test values more than $20 \%$ of the baseline values in more than two of the six cognitive function tests that were performed.

Results: In the cognitive function tests, there were no significant differences in the results obtained during the preoperative and postoperative periods for all tests and there were no mean differences observed in the preoperative and postoperative scores. The incidences of postoperative cognitive dysfunction in the control and rIPC groups were $28.6 \%(10$ patients) and 31.4\% (11 patients), respectively.

Conclusions: rIPC did not reduce the incidence of postoperative cognitive dysfunction after OPCAB surgery during the immediate postoperative period. (Korean J Anesthesiol 2013; 65: 418-424)

Key Words: Cognitive disorders, Neuroprotective effect, Off-pump coronary artery bypass.

Received: January 18, 2013. Revised: 1st, February 15, 2013; 2nd, April 22, 2013. Accepted: April 30, 2013.

Corresponding author: In-Cheol Choi, M.D., Ph.D., Department of Anesthesiology and Pain Medicine, Asan Medical Center, University of Ulsan College of Medicine, 388-1, Pungnap 2-dong, Songpa-gu, Seoul 138-736, Korea. Tel: 82-2-3010-3862, Fax: 82-2-470-1363, E-mail: icchoi@amc.seoul.kr (c) This is an open-access article distributed under the terms of the Creative Commons Attribution Non-Commercial License (http:// creativecommons.org/licenses/by-nc/3.0/), which permits unrestricted non-commercial use, distribution, and reproduction in any medium, provided the original work is properly cited. 


\section{Introduction}

Postoperative cognitive dysfunction is one of the neurological complications occurring after coronary artery bypass graft surgery ( $\mathrm{CABG}$ ), and the incidence of postoperative cognitive dysfunction after CABG is reported to be from 20 to $60 \%$ [1-3]. It has been suggested that the most important cause of postoperative cognitive dysfunction after cardiac surgery is the use of the cardiopulmonary bypass (CPB) system $[4,5]$. However, the incidence of postoperative cognitive dysfunction after off-pump coronary artery bypass graft (OPCAB) and CABG does not differ [6] as even the OPCAB does not use the CPB system. Therefore, not only CABG but also OPCAB requires some particular strategies in order to reduce the development of postoperative cognitive dysfunction.

The remote ischemic preconditioning (rIPC) is brief episodes of ischemia which may induce a patient's tolerance of a subsequent severe episode of ischemia, and several studies using animal models have shown that rIPC has cardioprotective and neuroprotective effects [7-9]. Moreover, some recent randomized controlled trials in human subjects have reported that rIPC improves the cardiac outcome after cardiac surgery with or without using the CPB system [10,11]. However, a randomized controlled trial in human subjects regarding the neuroprotective effect of rIPC after cardiac surgery has not yet been reported. Therefore, we performed this pilot study in order to determine whether rIPC reduced the occurrence of postoperative cognitive dysfunction in patients treated with an OPCAB.

\section{Materials and Methods}

This study protocol was approved by our institutional review board and the approval number was 2009-0374. All included patients signed written informed consent. Between October 10, 2009 and October 31, 2011 the patients who were scheduled to undergo elective OPCAB surgery at our institution enrolled in this pilot study. Among these patients, who underwent emergency, and were below the age of 40 or above the age of 80 , who had supported mechanical assistance devices (i.e. intra-aortic balloon pump, ventricular assist devices, extracorporeal membrane oxygenation), during the preoperative periods, were using inotropics preoperatively, had severe left ventricular dysfunction (ejection fraction $<30 \%$ ), and with a history of neuropsychiatric disease, were excluded. And we also excluded the patient who did not want to participate in the study any periods.

We collected demographic data such as, patient age, sex, height, weight, left ventricle ejection fraction (LVEF), euroscore, educational duration, history of diabetes mellitus, peripheral vascular disease, hypertension and smoking. The collected surgical and anesthetic factors were the number of anastomosis, surgery time, anesthesia administration time, anastomosis time and amount of packed red blood cell ( $\mathrm{pRBC}$ ) transfusion. The outcome variables were included the development of postoperative cognitive dysfunction, extubation time (the time interval between the end of surgery and extubation in intensive care unit), maximal cardiovascular component of the sequential organ failure assessment (SOFAc) score and the duration of their intensive care unit stay.

To define the baseline values of cognitive function, all patients were underwent cognitive function tests one day before surgery. The cognitive function tests in this study were composed of six categories, which recommended by the society of thoracic surgeons group for evaluation of neurobehavioral outcome after cardiac surgery [12]: (1) Seoul Verbal Learning Test (SVLT), (2) digit span forward test, (3) digit span backward test, (4) trailmaking test part A, (5) trail-making test part B, and (6) digit symbol substitution test (DSST) (Fig. 1). The SVLT is a relatively brief test of verbal learning and memory and is used to examine dementia patients when a more comprehensive memory assessment is not feasible or when serial testing is desired. The digit span forward and backward test attempts to measure short term memory. The trail-making test, the patient is asked to draw lines to connect numbers in ascending order. In Part $\mathrm{B}$, the circles include both numbers (1-13) and letters (A-L); in Part A, the patient draws lines to connect circles in an ascending pattern, but with the added task of alternating between the numbers and letters, i.e. 1-A-2-B-3-C, etc.. DSST is a neuropsychological test which assesses brain damage, dementia and depression.

The enrolled patients were divided into control and rIPC groups using a computer-generated randomization table. All patients were anesthetized following our institution's perioperative anesthesia management strategies for OPCAB surgery, and which were previously described in detail in another study [13]. All patients were induced anesthesia with an intravenous (IV) injection of $0.2 \mathrm{mg} / \mathrm{kg}$ etomidate and continuous infusion of propofol and remifentanil using a target control infusion (TCI) pump (Orchestra ${ }^{\circledR}$ Base Prima; Fresenius Kabi, Brezins, France). Then a bolus IV injection of $0.8 \mathrm{mg} / \mathrm{kg}$ rocuronium was administered for muscle relaxation to orotracheal intubation. To maintain the anesthesia during the intraoperative period, we infused propofol and remifentanil using a TCI pump with continuous infusion. To monitor anesthetic depth, we used bispectral index (BIS) monitor (A-2000, Aspect Medical Systems, Newton, MA, USA) and to maintain the BIS value between 40 to 60 , effect site concentration of propofol was titrated using a TCI pump. Effect site concentration of remifentanil was adjusted to maintain the heart rate and blood pressure within $20 \%$ of preoperative values. If mean arterial blood pressure and/or cardiac index decreased more than $20 \%$ of preoperative values when displacement of heart for distal anastomosis, vasoconstrictor or inotropics such 
A

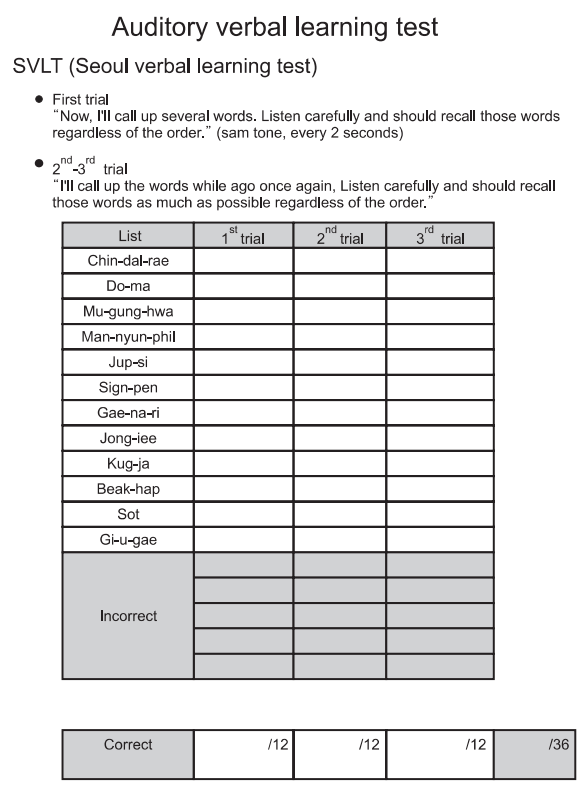

D

Digit symbol substitution test

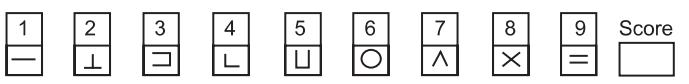

B \begin{tabular}{|l|l|l|l|l|l|l|l|l|l|l|l|l|l|l|l|l|l|l|l|l|l|l|l|l|}
\hline 1 & 5 & 4 & 2 & 7 & 6 & 3 & 5 & 7 & 2 & 8 & 5 & 4 & 6 & 3 & 7 & 2 & 8 & 1 & 9 & 5 & 8 & 4 & 7 & 3 \\
\hline
\end{tabular} \begin{tabular}{|l|l|l|l|l|l|l|l|l|l|l|l|l|l|l|l|l|l|l|l|l|l|l|l|l|}
\hline 6 & 2 & 5 & 1 & 9 & 2 & 8 & 3 & 7 & 4 & 6 & 5 & 9 & 4 & 8 & 3 & 7 & 2 & 6 & 1 & 5 & 4 & 6 & 3 & 7 \\
\hline
\end{tabular} \begin{tabular}{|l|l|l|l|l|l|l|l|l|l|l|l|l|l|l|l|l|l|l|l|l|l|l|l|l|}
\hline 9 & 2 & 8 & 1 & 7 & 9 & 4 & 6 & 8 & 5 & 9 & 7 & 1 & 8 & 5 & 2 & 9 & 4 & 8 & 6 & 3 & 7 & 9 & 8 & 6 \\
\hline
\end{tabular}

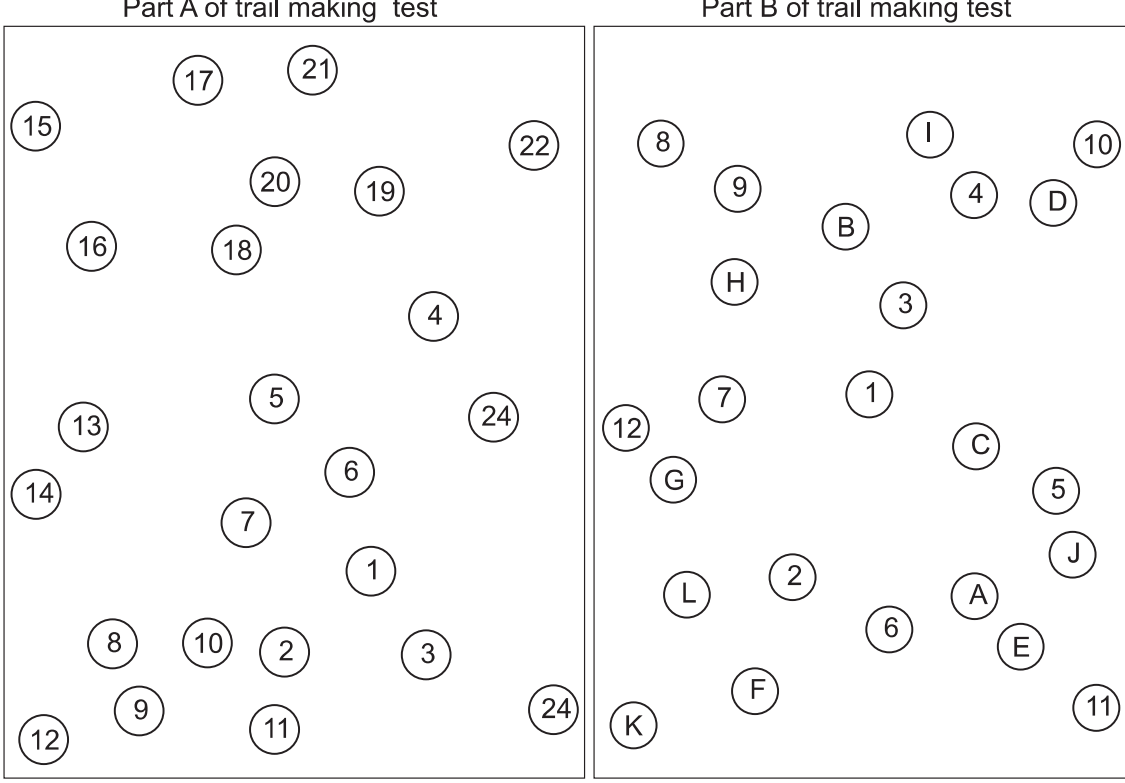

E

\begin{tabular}{|c|c|c|c|c|c|c|}
\hline \multicolumn{7}{|c|}{ Digit span } \\
\hline \multicolumn{2}{|c|}{ Foward } & \multirow{2}{*}{$(0,1,2)$} & \multicolumn{2}{|r|}{ Backward } & & $(0,1,2)$ \\
\hline \multirow{2}{*}{1.} & $5-8-2$ & & \multirow{2}{*}{1.} & $2-4$ & & \\
\hline & $6-9-4$ & & & $5-8$ & & \\
\hline \multirow{2}{*}{2.} & $6-4-3-9$ & & \multirow{2}{*}{2.} & $6-2-9$ & & \\
\hline & $7-2-8-6$ & & & $4-1-5$ & & \\
\hline \multirow{2}{*}{3.} & $4-2-7-3-1$ & & \multirow{2}{*}{3.} & $3-2-7-9$ & & \\
\hline & $7-5-8-3-6$ & & & $4-9-6-8$ & & \\
\hline \multirow{2}{*}{4.} & $6-1-9-4-7-3$ & & \multirow{2}{*}{4.} & $1-5-2-8-6$ & & \\
\hline & $3-9-2-4-8-7$ & & & $6-1-8-4-3$ & & \\
\hline \multirow{2}{*}{5.} & $5-9-1-7-4-2-8$ & & \multirow{2}{*}{5.} & $5-3-9-4-1-8$ & & \\
\hline & $4-1-7-9-3-8-6$ & & & $7-2-4-8-5-6$ & & \\
\hline \multirow{2}{*}{6.} & $5-8-1-9-2-6-4-7$ & & \multirow[b]{2}{*}{6.} & \begin{tabular}{|c|}
$8-1-2-9-3-6-5$ \\
\end{tabular} & & \\
\hline & $3-8-2-9-5-1-7-4$ & & & $4-7-3-9-1-2-8$ & & \\
\hline \multirow{2}{*}{7.} & $2-7-5-8-6-2-5-8-4$ & & \multirow[b]{2}{*}{7.} & $9-4-3-7-6-2-5-8$ & & \\
\hline & $7-1-2-9-4-2-5-6-8$ & & & $7-2-8-1-9-6-5-3$ & & \\
\hline & & & & & tal & \\
\hline
\end{tabular}

Fig. 1. The six categories of cognitive function test.

as phenylephrine, dopamine, or norepinephrine was administered. In the rIPC group, the application of rIPC was made during four, five-minute cycles of ischemia with five-minutes of reperfusion on the upper limb using a blood pressure cuff inflated to $200 \mathrm{mmHg}$ before coronary artery anastomosis, however, the control group only had the blood pressure cuff placed on an upper limb. The other anesthetic and surgical management components did not different between the two groups. Cell salvage device was used for all patients and salvaged blood was reinfused all patients. Packed red blood cell was transfused when serum hemoglobin concentration less than $8 \mathrm{~g} / \mathrm{dl}$. We checked core temperature by pulmonary arterial catheter intraoperative periods and kept above $36^{\circ} \mathrm{C}$ using a forced-air warming system. We also used cerebral oxymeter (Invos system, Somanetics
Corporation, Troy, Michigan, USA) for monitoring of cerebral perfusion state. All anesthetic drugs were discontinued at the end of surgery and all patients were transferred to the intensive care unit immediately after surgery. Seven days after the surgery, postoperative cognitive function tests were performed in order to define the development of postoperative cognitive dysfunction. The cognitive function tests were performed by one clinical research assistant who well-educated about the cognitive function tests in both periods. The research assistant did not know to which group the patient had allocated.

The continuous variables in this study were summarized as the mean \pm SD or medians with range and the categorical variables were frequency and percentages. Continuous data were analyzed using the paired t-test or the Mann-Whitney rank sum 


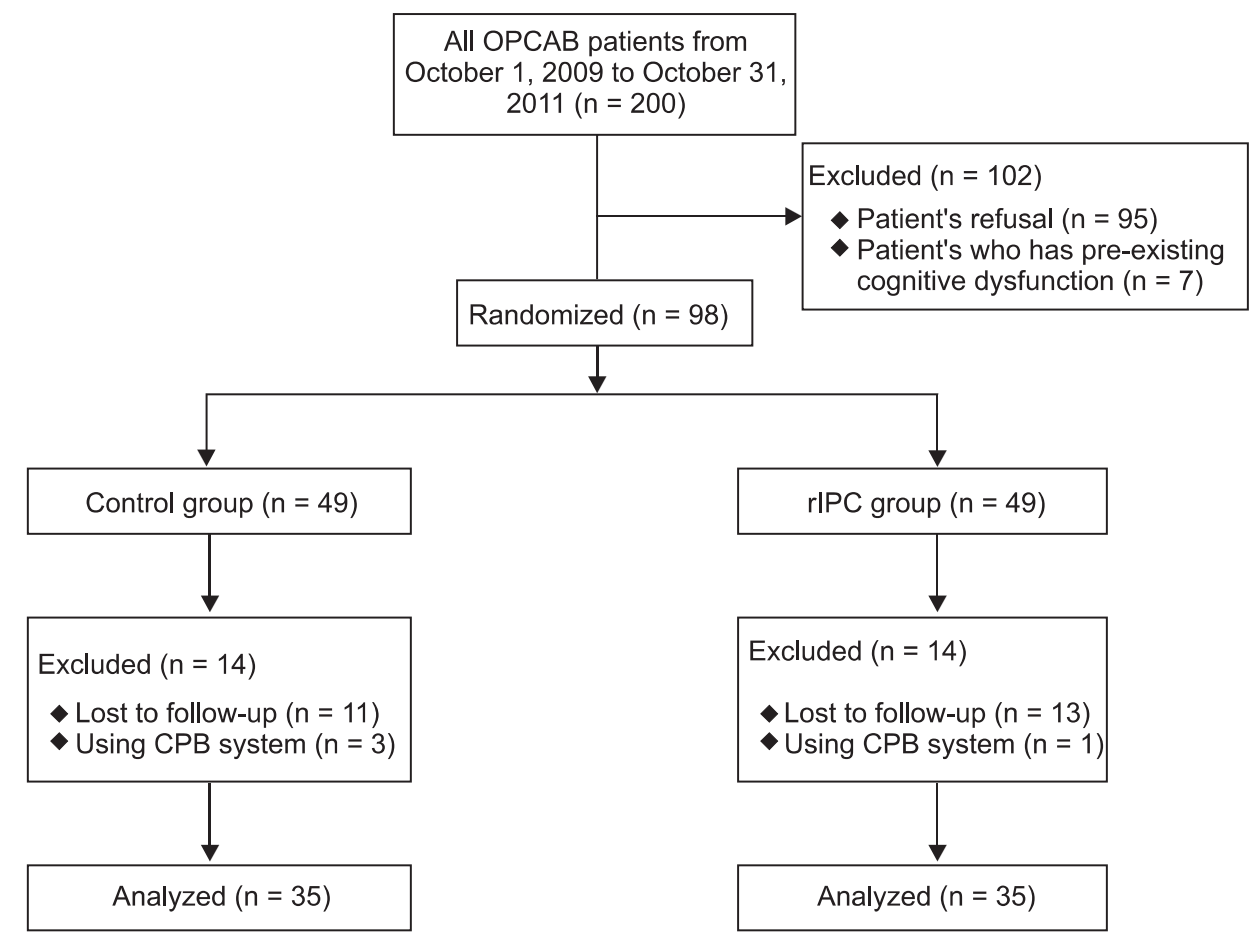

Fig. 2. Flow of patients throughout the trial.
U test, and categorical data were tested using the Pearson's $\chi^{2}$ test or the Fisher's exact test. We defined postoperative cognitive dysfunction according to the decreased postoperative test values of more than $20 \%$ from the baseline values in more than two tests of the six cognitive function tests we performed. The P values were reported as two-sided, and the statistically significant value was $<0.05$. All statistical analysis in this pilot study used SPSS 14.0 software (SPSS, Chicago, IL, USA) and SigmaPlot 12.0 software (Systat, San Jose, CA, USA)

\section{Results}

Two hundred patients underwent OPCAB surgery at our medical institution. Only 98 patients were enrolled in this study either because of their refusal (95 patients) or a pre-existing cognitive dysfunction (seven patients). Among the enrolled patients, 98 completed the preoperative cognitive function test. Ninety-four patients successfully underwent OPCAB and four patients were converted to CABG. Three of the rIPC group patients and one of the control group patients were converted to CABG. The reasons for their conversion included hemodynamic instability (two patients), anatomic problem of coronary arteries (one patient) or both (one patient). Moreover, 11 of the rIPC group patients and 13 of the control group patients did not complete the postoperative cognitive function test as they chose to drop out of the study. Therefore, seventy patients were included in the study and the final analysis (Fig. 2).

In the final analysis, the control group (C) consisted of 35 pa-
Table 1. Baseline Characteristics and Perioperative Characteristics of Patients

\begin{tabular}{lccc}
\hline & $\begin{array}{c}\text { Control } \\
(\mathrm{n}=35)\end{array}$ & $\begin{array}{c}\text { rIPC } \\
(\mathrm{n}=35)\end{array}$ & P value \\
\hline Demographic data & & & \\
Age (yr) & $59.0(8.3)$ & $61.1(7.3)$ & 0.28 \\
Sex (M/F) & $27 / 8$ & $30 / 5$ & 0.75 \\
Height (cm) & $164.9(8.6)$ & $164.2(7.7)$ & 0.74 \\
Weight (kg) & $67.6(10.1)$ & $66.16(7.7)$ & 0.50 \\
LVEF (\%) & $57.8(10.5)$ & $57.3(9.7)$ & 0.82 \\
EuroSCORE (\%) & $2.1(1.6)$ & $2.1(1.4)$ & 0.97 \\
Education year (yr) & $11.8(4.0)$ & $12.0(3.7)$ & 0.83 \\
Diabetes mellitus & $10(28.6 \%)$ & $17(48.6 \%)$ & 0.14 \\
Hypertension & $21(60.0 \%)$ & $22(62.9 \%)$ & 1.00 \\
Smoking & $22(62.9 \%)$ & $16(45.7 \%)$ & 0.23 \\
Peripheral vascular disease & $5(14.3 \%)$ & $4(11.4 \%)$ & 1.00 \\
Preoperative medication & & & \\
ACEI or ARB & $6(17.1 \%)$ & $10(28.6 \%)$ & 0.39 \\
Calcium channel blocker & $14(40.0 \%)$ & $19(54.3 \%)$ & 0.34 \\
Beta-blocker & $16(45.7 \%)$ & $20(57.1 \%)$ & 0.47 \\
Lipid-lowering agent & $13(37.1 \%)$ & $13(37.1 \%)$ & 1.00 \\
Intra- and Postoperative & & & \\
characteristics & & & \\
Number of anastomosis & $2.9(1.0)$ & $2.8(0.8)$ & 0.79 \\
Operation time (min) & $243.3(63.4)$ & $255.8(56.6)$ & 0.39 \\
Anesthesia time (min) & $280.6(58.1)$ & $292.0(55.9)$ & 0.41 \\
pRBC transfusion (U) & $0.74(1.0)$ & $0.46(0.9)$ & 0.18 \\
Anastomosis time (min) & $63.0(20.8)$ & $60.9(17.8)$ & 0.30 \\
Extubation time (hr) & $13.2(5.1)$ & $12.5(6.8)$ & 0.89 \\
Maximal SOFAc score > 1 & $26(74.3 \%)$ & $28(80.0 \%)$ & 0.78 \\
ICU stay (hr) & $56.1(26.8)$ & $55.3(19.6)$ & 0.64 \\
\hline
\end{tabular}

rIPC: remote ischemic preconditioning, LVEF: left ventricle ejection fraction, EuroSCORE: European System for Cardiac Operative Risk Evaluation, ACEI: angiotensin-converting enzyme inhibitor, ARB: angiotensin receptor blocker, pRBC: packed red blood cell, SOFAc: cardiovascular sequential organ failure assessment in the first 24 hours, ICU: intensive care unit. 
Table 2. Mean Preoperative and Postoperative Scores for All Tests

\begin{tabular}{|c|c|c|c|c|}
\hline \multirow{2}{*}{ Test } & \multicolumn{2}{|c|}{ Mean preoperative scores } & \multicolumn{2}{|c|}{ Mean postoperative scores } \\
\hline & Control $(\mathrm{n}=35)$ & $\operatorname{rIPC}(\mathrm{n}=35)$ & Control $(\mathrm{n}=35)$ & $\operatorname{rIPC}(\mathrm{n}=35)$ \\
\hline Seoul verbal learning test (score) & $19.0(5.2)$ & $18.7(5.5)$ & $22.9(6.2)$ & $22.9(5.6)$ \\
\hline Part Aof trail making test (sec) & $53.2(32.5)$ & $49.1(14.8)$ & $53.1(33.6)$ & $54.7(26.7)$ \\
\hline Part B of trail making test $(\mathrm{sec})$ & $133.1(103.8)$ & $137.2(43.9)$ & $149.8(128.4)$ & $147.0(53.4)$ \\
\hline Digit symbol substitution test (score) & $40.7(13.9)$ & $42.5(11.5)$ & $40.1(15.1)$ & $41.2(11.1)$ \\
\hline Digit span forward test (score) & $9.5(2.7)$ & $8.2(2.7)$ & $10.1(2.8)$ & $8.4(2.6)$ \\
\hline Digit span backward test (score) & $6.4(2.7)$ & $5.2(2.2)$ & $6.0(3.0)$ & $5.3(2.0)$ \\
\hline
\end{tabular}

rIPC: remote ischemic preconditioning.

Table 3. Mean Difference of the Preoperative and Postoperative Scores for Each Test and Number of Patients Who Decreased Postoperative Cognitive Function

\begin{tabular}{|c|c|c|c|c|}
\hline \multirow[t]{2}{*}{ Test } & \multicolumn{2}{|c|}{ Mean difference seven days postoperatively } & \multicolumn{2}{|c|}{$\begin{array}{l}\text { Number of patients who decreased } \\
\text { postoperative cognitive function test }\end{array}$} \\
\hline & Control $(\mathrm{n}=35)$ & $\operatorname{rIPC}(\mathrm{n}=35)$ & Control $(\mathrm{n}=35)$ & $\operatorname{rIPC}(\mathrm{n}=35)$ \\
\hline Seoul verbal learning test (score) & $3.9(5.3)$ & $4.3(5.2)$ & $2(5.7 \%)$ & $1(2.9 \%)$ \\
\hline Part A of trail making test ( $\mathrm{sec}$ ) & $-0.1(18.0)$ & $5.6(21.7)$ & $10(28.6 \%)$ & $8(22.9 \%)$ \\
\hline Part B of trail making test (sec) & $16.7(54.5)$ & $9.8(41.4)$ & $10(28.6 \%)$ & $9(25.7 \%)$ \\
\hline Digit symbol substitution test (score) & $-0.6(7.1)$ & $-1.3(4.2)$ & $4(11.4 \%)$ & $1(2.9 \%)$ \\
\hline Digit span forward test (score) & $0.6(1.4)$ & $0.2(1.6)$ & $5(14.3 \%)$ & $7(20.0 \%)$ \\
\hline Digit span backward test (score) & $-0.4(1.7)$ & $0.1(2.0)$ & $13(37.1 \%)$ & $9(25.7 \%)$ \\
\hline
\end{tabular}

rIPC: remote ischemic preconditioning.

tients and the rIPC group (R) of 35 patients. In these two groups, there were no differences in either the baseline characteristics or the perioperative characteristics (Table 1). In the cognitive function tests, there were no significant differences seen during the preoperative and postoperative periods in any of the tests (Table 2). In addition, the mean differences of the preoperative and postoperative scores did not observe either (Table 3 ). The incidence of postoperative cognitive dysfunction in the control and rIPC group patients was 28.6\% (10 patients) and 31.4\% (11 patients), respectively $(\mathrm{P}=0.794)$. Additionally, there were no symptomatic stroke and death in both groups.

\section{Discussion}

In this pilot study, the incidence of postoperative cognitive dysfunction of the control and rIPC groups was 28.6\% (10 patients) and $31.4 \%$ (11 patients), respectively. Unfortunately, this result indicates that the rIPC before coronary artery anastomosis does not reduce the development of postoperative cognitive dysfunction after OPCAB surgery.

Postoperative cognitive dysfunction is a relatively common complication and increases the overall cost, length of hospital stay, morbidity, and mortality after cardiac surgery. The underlying mechanisms of the development of postoperative cognitive dysfunction after cardiac surgery are unclear, with the possible causes being cerebral microemboli, systemic inflammatory response, cerebral hypoperfusion and/or ischemic/reperfusion damage and hormonal changes [14]. Among these causes, the most common is cerebral microemboli due to the use of the $\mathrm{CPB}$ system. However, when anastomosis of the coronary artery is performed at the posterior wall of the heart, other factors, such as cerebral hypoperfusion and ischemic/reperfusion damages in the OPCAB surgery performed without the CPB system may be more important rather than cerebral microemboli [15].

Although many trials have been performed to reduce the development of postoperative cognitive dysfunction, the definitive strategies have not yet been established. Some recent studies have reported that the rIPC has an organ protective effect, including the brain in both humans and in animal models $[8,16,17]$. Although the exact mechanisms of the neuroprotective effect of rIPC are unclear, the ischemic tolerance induced by anti-excitotoxicity, anti-apoptosis, anti-inflammation, protection of mitochondria and increased anti-oxidant mechanisms is thought be have an important role in the neuroprotective effects of rIPC [18]. Several animal studies have shown that rIPC has a neuroprotective effect in an ischemic model. For example, Wei et al. showed that rIPC has a long term neuroprotective effect in a focal ischemia model by inhibiting the activities of the galectin-9/Tim-3 pathway, iNOS, and nitrotyrosine [19]. However, some animal studies have reported that the rIPC did not have neuroprotective effects. For example, Saxena et al. [20] demonstrated that hippocampal CA1 neuronal cell death in rats 
was not protected by rIPC. Regrettably, we did not observe a difference in the incidence of postoperative cognitive dysfunction regardless of the applications of rIPC before coronary artery anastomosis during OPCAB surgery in our pilot study. The discrepancy of the protective effect of rIPC for animal and human studies has also has been reported in other organs. For example, Przyklenk et al. [9] showed that rIPC has a cardiac protective effect in dogs. However, Rahman et al. [21] reported that rIPC did not reduce troponin release or improve the hemodynamics in their prospective, randomized, placebo-controlled trial in a human population. As mentioned earlier, the underlying mechanisms of postoperative cognitive dysfunction involve many factors such as ischemic/reperfusion injury, hormonal change, and inflammatory mediators $[22,23]$. rIPC alone may not affect all of the involved factors in the development of postoperative cognitive dysfunction. Furthermore, the possible neuroprotective role of rIPC may not be critical in the development of postoperative cognitive dysfunction in patients undergoing OPCAB.

In addition, the duration of application of rIPC may affect the current results. Ren et al. [24] showed that the application of three, 15-minute cycles of, but not of three, five-minute cycles, for limb rIPC reduced the infarct size in focal cerebral ischemia of rats. In our study protocol, rIPC was applied in four, fiveminute cycles before coronary artery anastomosis, which might be not enough to decrease postoperative cognitive dysfunction. Moreover, the time point of the application of rIPC affected our results. For example, more reduced infarct size was observed by remote ischemic per-conditioning (four, five-minute cycles) during middle cerebral artery occlusion than immediately be- fore ischemic insult in a rat model of focal ischemia [25]. This means that the time point of application of rIPC may be critical for neuroprotection. Application of rIPC before coronary artery anastomosis in our study may be a less appropriate time point in order to reduce the development of postoperative cognitive dysfunction.

Our pilot study has some limitations. First, only 70 patients remained in the final analysis in our pilot study. This is a relatively small sample size to determine the difference of development of postoperative cognitive dysfunction between two groups. Moreover, as the drop-out rate of our pilot study was 20\% (28 of 98 patients). This may constitute a selection bias. In addition, the long term effect of rIPC on cognitive dysfunction may different in our results, as we performed postoperative cognitive function tests only at POD 7. Therefore, we believe that some large-scale, prospective studies including long-term follow-up results are required. Second, we described earlier, cerebral hypoperfusion and ischemic/reperfusion damages when anastomosis of the coronary artery is performed at the posterior wall of the heart were important to development of postoperative cognitive dysfunction. In this study, we did not collect data such as mixed venous saturation, cardiac index, and cerebral oxymeter data in that period. However, we believe that intraoperative characteristics such as anastomosis time, number of graft and pRBC transfusion were also reflected the anesthetic and surgical risk for development of postoperative cognitive dysfunction.

In conclusion, rIPC did not reduce the incidence of postoperative cognitive dysfunction after OPCAB surgery during the immediate postoperative period.

\section{References}

1. Mahanna EP, Blumenthal JA, White WD, Croughwell ND, Clancy CP, Smith LR, et al. Defining neuropsychological dysfunction after coronary artery bypass grafting. Ann Thorac Surg 1996; 61: 1342-7.

2. Newman MF, Kirchner JL, Phillips-Bute B, Gaver V, Grocott H, Jones RH, et al. Longitudinal assessment of neurocognitive function after coronary-artery bypass surgery. N Engl J Med 2001; 344: 395-402.

3. Newman SP, Harrison MJ. Coronary-artery bypass surgery and the brain: persisting concerns. Lancet Neurol 2002; 1: 119-25.

4. Newman MF, Mathew JP, Grocott HP, Mackensen GB, Monk T, Welsh-Bohmer KA, et al. Central nervous system injury associated with cardiac surgery. Lancet 2006; 368: 694-703.

5. Selnes OA, Goldsborough MA, Borowicz LM, McKhann GM. Neurobehavioural sequelae of cardiopulmonary bypass. Lancet 1999; 353 : 1601-6.

6. Van Dijk D, Jansen EW, Hijman R, Nierich AP, Diephuis JC, Moons KG, et al. Cognitive outcome after off-pump and on-pump coronary artery bypass graft surgery: a randomized trial. JAMA 2002; 287: 1405-12.

7. Ren C, Gao X, Steinberg GK, Zhao H. Limb remote-preconditioning protects against focal ischemia in rats and contradicts the dogma of therapeutic time windows for preconditioning. Neuroscience 2008; 151: 1099-103.

8. Dave KR, Saul I, Prado R, Busto R, Perez-Pinzon MA. Remote organ ischemic preconditioning protect brain from ischemic damage following asphyxial cardiac arrest. Neurosci Lett 2006; 404: 170-5.

9. Przyklenk K, Bauer B, Ovize M, Kloner RA, Whittaker P. Regional ischemic 'preconditioning' protects remote virgin myocardium from subsequent sustained coronary occlusion. Circulation 1993; 87: 893-9.

10. Cheung MM, Kharbanda RK, Konstantinov IE, Shimizu M, Frndova H, Li J, et al. Randomized controlled trial of the effects of remote ischemic preconditioning on children undergoing cardiac surgery: first clinical application in humans. J Am Coll Cardiol 2006; 47: $2277-82$. 
11. Hong DM, Jeon Y, Lee CS, Kim HJ, Lee JM, Bahk JH, et al. Effects of remote ischemic preconditioning with postconditioning in patients undergoing off-pump coronary artery bypass surgery--randomized controlled trial. Circ J 2012; 76: 884-90.

12. Murkin JM, Newman SP, Stump DA, Blumenthal JA. Statement of consensus on assessment of neurobehavioral outcomes after cardiacsurgery. Ann Thorac Surg 1995; 59: 1289-95.

13. Lee EH, Yun SC, Chin JH, Choi DK, Son HJ, Kim WC, et al. Prognostic implications of preoperative E/e' ratio in patients with off-pump coronary artery surgery. Anesthesiology 2012; 116: 362-71.

14. Hogue CW Jr, Palin CA, Arrowsmith JE. Cardiopulmonary bypass management and neurologic outcomes: an evidence-based appraisal of current practices. Anesth Analg 2006; 103: 21-37.

15. Liu YH, Wang DX, Li LH, Wu XM, Shan GJ, Su Y, et al. The effects of cardiopulmonary bypass on the number of cerebral microemboli and the incidence of cognitive dysfunction after coronary artery bypass graft surgery. Anesth Analg 2009; 109: 1013-22.

16. Xu T, Gong Z, Zhu WZ, Wang JF, Li B, Chen F, et al. Remote ischemic preconditioning protects neurocognitive function of rats following cerebral hypoperfusion. Med Sci Monit 2011; 17: BR299-304.

17. Lazaris AM, Maheras AN, Vasdekis SN, Karkaletsis KG, Charalambopoulos A, Kakisis JD, et al. Protective effect of remote ischemic preconditioning in renal ischemia/reperfusion injury, in a model of thoracoabdominal aorta approach. J Surg Res 2009; 154: 267-73.

18. Gidday JM. Cerebral preconditioning and ischaemic tolerance. Nat Rev Neurosci 2006; 7: 437-48.

19. Wei D, Ren C, Chen X, Zhao H. The chronic protective effects of limb remote preconditioning and the underlying mechanisms involved in inflammatory factors in rat stroke. PLoS One 2012; 7: e30892.

20. Saxena P, Bala A, Campbell K, Meloni B, d'Udekem Y, Konstantinov IE. Does remote ischemic preconditioning prevent delayed hippocampal neuronal death following transient global cerebral ischemia in rats? Perfusion 2009; 24: 207-11.

21. Rahman IA, Mascaro JG, Steeds RP, Frenneaux MP, Nightingale P, Gosling P, et al. Remote ischemic preconditioning in human coronary artery bypass surgery: from promise to disappointment? Circulation 2010; 122(11 Suppl): S53-9.

22. Bhuiyan MI, Kim YJ. Mechanisms and prospects of ischemic tolerance induced by cerebral preconditioning. Int Neurourol J 2010; 14: 20312.

23. Funder KS, Steinmetz J, Rasmussen LS. Cognitive dysfunction after cardiovascular surgery. Minerva Anestesiol 2009; 75: 329-32.

24. Ren C, Gao X, Steinberg GK, Zhao H. Limb remote-preconditioning protects against focal ischemia in rats and contradicts the dogma of therapeutic time windows for preconditioning. Neuroscience 2008; 151: 1099-103.

25. Hahn CD, Manlhiot C, Schmidt MR, Nielsen TT, Redington AN. Remote ischemic per-conditioning a novel therapy for acute stroke? Stroke 2011; 42: 2960-2. 